\title{
Characterization of the geometry of an array of CCD pixel detectors
}

\author{
M. Trassinelli ${ }^{a}$, C. David ${ }^{b}$, D. Gotta ${ }^{c}$, M. Hennebach ${ }^{c}$, \\ P. Indelicato ${ }^{a}$, E.-O. Le Bigot ${ }^{a}$, N. Nelms ${ }^{d}$, L.M. Simons ${ }^{b}$ \\ ${ }^{a}$ Laboratoire Kastler Brossel, \\ École Normale Supérieure et Université Pierre et Marie Curie, \\ Case 74, 4 Place Jussieu, F-75005 Paris, France \\ ${ }^{b}$ Paul Scherrer Institut, Villigen PSI, Switzerland \\ ${ }^{c}$ Institut für Kernphysik, Forschungszentrum Jülich, \\ Jülich, Germany \\ $d$ Department of Physics and Astronomy, \\ University of Leicester, Leicester, United Kingdom
}

The pixel distance as well as the relative orientation of an array of 6 CCD pixel detector chips have been determined with accuracies of about $1 \mathrm{~nm}$ and $50 \mu \mathrm{rad}$, respectively. This accuracy satisfies the needs of modern crystal spectroscopy experiments in the field of exotic atoms. Two different measurements have been attempted by illuminating masks in front of the detector array by a remote source of radiation. In one case an aluminum mask was irradiated with $\mathrm{X}$-rays and in a second attempt a nanometric quartz wafer was illuminated by a light bulb. Both methods gave consistent results with a smaller error for the optical method. Furthermore, the thermal expansion with temperature of the CCDs could be determined.

\section{Introduction}

Charge coupled devices (CCDs) are ideally suited X-ray detectors in exotic atoms spectroscopy in the few $\mathrm{keV}$ range because of excellent energy resolution and the in build-in two-dimensional spatial information [1]. In particular, they can be used as detectors of focusing of crystal spectrometers to allow the investigation of the characteristic X-radiation from exotic atoms in the few keV range. 
The detector described in this work was set up in a spherically bent crystal spectrometer used in two ongoing experiments at the Paul-Scherrer-Institut: the measurements of the charged pion mass $[2,3]$ and the strong interaction shift and width of the pionic hydrogen ground state $[4,5]$. The detector is composed by an array of two vertical columns of 3 CCDs each [6]. Each device has $600 \times 600$ square pixels with a nominal dimension of $40 \mu \mathrm{m}$ at room temperature. Each pixel is defined by an open-electrode structure. For this reason, the dimension characterizing the detector is rather the distance between adjacent pixels than the pixel size.

As the CCD is usually operated at $-100^{\circ} \mathrm{C}$, the knowledge of the pixel distance at the working temperature is essential for crystal spectroscopy, because any angular difference is calculated from the measured position difference of X-ray reflections. Furthermore, for an array like the one being used here, the relative orientation of the CCD chips has to be known at the same level of accuracy than the average pixel distance.

A first attempt to determine the relative positions has been made using a wire eroded aluminum mask illuminated by sulfur fluorescence X-rays produced by means of an X-ray tube. The alignment of the mask pattern allowed to estimate the relative CCD position with an accuracy of about 0.05 pixel and the relative rotation to slightly better than $100 \mu \mathrm{rad}[7]$. The accuracy was limited by the quality of the Al mask. In order to reduce the error a new measurement was set-up by using a high-precision quartz wafer illuminating the CCD with light. This allowed in addition the measurement of the temperature dependence of the pixel distance.

\section{Measurement of the pixel distance and the relative orientation of the CCD chips.}

\subsection{Description of the set-up}

The quartz wafer itself is an optical cross grating manufactured by the Laboratory of Micro- and Nanotechnology of the Paul Scherrer Institut. The grating is $40 \mathrm{~mm}$ wide and $70 \mathrm{~mm}$ high. It is composed by vertical and horizontal lines being $50 \mu \mathrm{m}$ thick and separated by $2 \mathrm{~mm}$. The line pattern was defined by electron beam lithography using a laser interferometer controlled lithography system. The accuracy, i.e., its deviation from linearity, is of the order of $0.1 \mu \mathrm{m}$ over the full size. The wafer was positioned $37 \mathrm{~mm}$ in front of the CCD array and illuminated with short light pulses by a point-like light source located at a distance of $6426.7 \mathrm{~mm}$. During the measurements the wafer temperature was monitored to stay at room temperature. The integration time per picture was $10 \mathrm{~s}$ with the bulb shining for $6 \mathrm{~s}$ for each selected temperature. The temperature of the CCD was changed between $-105^{\circ} \mathrm{C}$ and $-40^{\circ} \mathrm{C}$.

\subsection{Measurement of the pixel distance}

For the determination of the pixel distance, a simultaneous linear fit of two adjacent lines was performed under the constraint that the two lines are parallel. When cutting out 

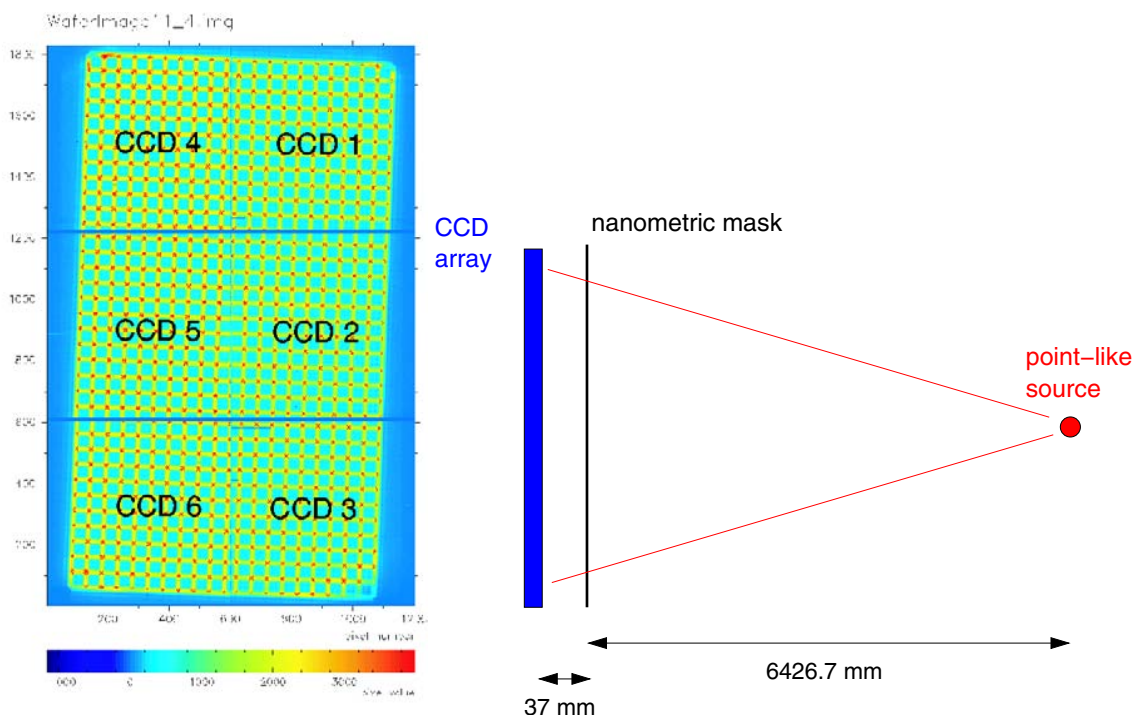

Figure 1: Left: Image of the quartz wafer as seen without correcting for the relative positions of the CCDs. Right: Scheme of the experiment set-up.

the crossing points the diffraction pattern is fit to a superposition of 5 Gaussian profiles (central peak, the first and second side maxima) and a background left and right.

For each CCD we obtained about 180 independent evaluations of the pixel distance coming from different line pairs. The average value of the pixel distance is obtained by a Gaussian fit to the histogram obtained from the individual values. Two series of images were available and both series yield consistent results. Therefore, the final value was calculated from the sum of the two distribution.

A priori, the pixel distance could be different for various CCDs due to fabrication uncertainties. This assumption was tested applying the Student's t-test [8] between distributions from different CCDs. The only significant difference in the distribution obtained is coming from CCD2 and CCD5. However, in these CCDs we observe a parasitic image of the mask, probably due to the reflection between the detector and the mask itself mentioned above. Therefore, the final value of the pixel distance is given by the weighted average of the individual CCD values excluding CCD2 and CCD5. Thus we obtain for the pixel distance:

Horizontal: $39.9764 \pm 0.0009 \mu m$

Vertical: $\quad 39.9779 \pm 0.0004 \mu m$. 


\subsection{Measurement the relative orientation of the chips}

A correct reconstruction of the two-dimensional image from the 6 CCDs requires the knowledge of the relative position of the pixels for all chips, i.e., the relative orientation (position and angular orientation).

For the relative position of the whole array, one CCD has to be chosen as a reference. The unambiguous calculation of the horizontal and vertical shift $(\Delta x$ and $\Delta y)$ and the orientation difference $(\Delta \Theta)$ between two frames requires the information coming from at least 4 lines: one pair of perpendicular lines in the reference CCD and one pair in any other CCD. Using the line position parameters, it is possible to build a function depending on $\Delta x, \Delta y$ and $\Delta \Theta$, which is minimal when the shift and rotation values are optimal. A set of values is obtained by minimization relative to line pairs randomly selected.

For any arbitrary choice of the line pairs, in principle different results of the position parameters may be obtained. Consequently, the final values of $\Delta x, \Delta y$ and $\Delta \Theta$ are given, as for the pixel distance, by a Gaussian fit to the distribution of the individual values. The final result for the CCD position uses two series of 10 images each. The precision obtained for each series is around 0.001 pixels for $\Delta x$ and $\Delta y$, and $3 \mu \mathrm{rad}$ for the relative rotation $\Delta \Theta$. The systematic errors were estimated by comparing the results between the two series of data acquisition. We obtain a total error of 0.02 pixels for $\Delta x$ and $\Delta y$, and $55 \mu \mathrm{rad}$ for $\Delta \Theta$. The final values for the shift and rotation of the CCDs are given in Table 1.

Table 1: CCD relative position and orientation with CCD2 as reference

\begin{tabular}{lrrr}
\hline CCD & $\Delta x$ (pixels) & $\Delta y$ (pixels) & $\Delta \Theta(\mathrm{rad})$ \\
\hline CCD2-CCD1 & $-1.245 \pm 0.020$ & $11.398 \pm 0.019$ & $-0.000595 \pm 0.000055$ \\
CCD2-CCD3 & $0.503 \pm 0.020$ & $-11.018 \pm 0.019$ & $-0.000698 \pm 0.000055$ \\
CCD2-CCD4 & $-12.850 \pm 0.020$ & $10.279 \pm 0.019$ & $0.000801 \pm 0.000055$ \\
CCD2-CCD5 & $-13.574 \pm 0.020$ & $1.742 \pm 0.019$ & $0.002194 \pm 0.000055$ \\
CCD2-CCD6 & $-15.967 \pm 0.020$ & $-9.432 \pm 0.019$ & $0.005530 \pm 0.000055$ \\
\hline
\end{tabular}

\subsection{Temperature dependence of the pixel distance}

For the determination of the temperature dependence images between $-105^{\circ} \mathrm{C}$ and $-40^{\circ} \mathrm{C}$ were acquired. For each condition the same analysis method as described in Sec. 2.2 was applied. As expected, the pixel distance increases with increasing temperature except for the vertical pixel distance at $-40^{\circ} \mathrm{C}$ (see Table 2). This effect maybe caused by the high noise level at this temperature. The average of the thermal expansion coefficient is obtained by a simple linear extrapolation of the data between $-105^{\circ} \mathrm{C}$ and $-60^{\circ} \mathrm{C}$. The results are: $2.8 \cdot 10^{-6} \pm 1.0 \cdot 10^{-6} K^{-1}$ for the horizontal distance and $1.3 \cdot 10^{-6} \pm 0.4 \cdot 10^{-6} K^{-1}$ for the vertical. These values are in the range of the thermal expansion coefficient of silicon for the temperatures considered $\left(0.8-1.6 \cdot 10^{-6} K^{-1}[9]\right)$. 
Table 2: Pixel distance values at different detector temperatures.

\begin{tabular}{rrr}
\hline Temp. $\left({ }^{\circ} \mathrm{C}\right)$ & Hor. pixel dist. $(\mu \mathrm{m})$ & Vert. pixel dist. $(\mu \mathrm{m})$ \\
\hline-105 & $39.9796 \pm 0.0014$ & $39.9779 \pm 0.0006$ \\
-100 & $39.9764 \pm 0.0009$ & $39.9779 \pm 0.0004$ \\
-80 & $39.9796 \pm 0.0020$ & $39.9794 \pm 0.0006$ \\
-60 & $39.9827 \pm 0.0017$ & $39.9800 \pm 0.0006$ \\
-40 & $39.9837 \pm 0.0013$ & $39.9762 \pm 0.0010$ \\
\hline
\end{tabular}

\section{Conclusion}

We have demonstrated that the average pixel distance on a CCD chip can be determined with an accuracy of better than $25 \mathrm{ppm}$ : $39.9764 \pm 0.0009 \mu \mathrm{m}$ for the horizontal pixel distance and $39.9779 \pm 0.0004 \mu \mathrm{m}$ for the vertical pixel distance. Moreover the temperature dependence of the pixel distance was studied. The relative rotations and positions of the individual CCD chips have been measured to a precision of $55 \mu \mathrm{rad}$ and 0.02 pixel, respectively. The accuracy achieved satisfies the requirements of a recent attempt to measure the charged pion mass.

\section{References}

[1] D. Gotta. Precision spectroscopy of light exotic atoms. Prog. Part. Nucl. Phys., 52:133-195, 2004.

[2] Pion Mass Collaboration. Measurement Of The Pion Mass. PSI experiment proposal R-97.02, 1997.

[3] N. et al Nelms. Precision Determination of Pion Mass Using X-Ray Ccd Spectroscopy. Nucl. Instr. Meth. A, 477(1-3):461-8, 2002.

[4] Pionic Hydrogen Collaboration. Measurement of the Strong Interaction Width and Shift of the Ground State of Pionic Hydrogen. PSI experiment proposal R-98.01, 1998.

[5] D. F. et al Anagnostopoulos. Precision measurements in pionic hydrogen. Nucl. Phys. A, 721:849-852, 2003.

[6] N. et al Nelms. A Large Area Ccd X-Ray Detector for Exotic Atom Spectroscopy. Nucl. Instrum. Meth. A, 484(1-3):419-31, 2002.

[7] M. Hennebach. Precision Measurement of Ground State Transition in Pionic Hydrogen. PhD thesis, Universität zu Köln, Köln, 2004.

[8] W. H. Press, S. A. Teukolsky, Vetterling W. T., and B. P. Flannery. Numerical Recipes in Fortran 77: The Art of Scientific Computing. Cambridge University Press, New York, second edition, 2001.

[9] K. G. Lyon, G. L. Salinger, C. A. Swenson, and G. K. White. Linear thermal expansion measurements on silicon from 6 to 340 k. J. Appl. Phys., 48(3):865-868, 1977. 
